\title{
Griginal article
}

\section{Usefulness of C-reactive protein in diagnosis of intrapartum and postpartum neonatal sepsis \\ Khaled Amro}

\begin{abstract}
:
Objective:

To determine effects of intrapartum risk factors for early onset sepsis (EOS) on CRP levels in neonates and to assess the suitability of this test in diagnosing EOS.

Design: Cohort study.

Setting:

Labour and post natal wards in a pediatric and obstetric department at military hospital in Zarka.
\end{abstract}

\section{Subjects:}

200 neonates at risk of developing infection.

\section{Methods:}

CRP levels in cord blood and neonatal blood at 24 hrs were estimated using commercial kits. Babies were observed for signs of sepsis for at least 48 hours.

\section{Results:}

Seven (3.5\%) neonates had elevated CRP levels in the cord blood. At 24 hours, 82 (41\%) babies had elevated levels. Elevated cord CRP levels was significantly associated with rupture of membranes for 24 hours labour more than 12 hours, and maternal fever. At 24 hours, elevated CRP levels were associated with primiparity, more than three vaginal examinations after membrane rupture, meconium staining of amniotic fluid and amnioinfusion. Ten (4\%) babies developed EOS. The negative predictive value for elevated CRP levels at $24 \mathrm{~h}$ was $99 \%$.

\section{Conclusion:}

Several intrapartum risk factors for EOS can cause elevation in CRP levels. However, this test may be useful in excluding infection.

Keywords: C-reactive protein, neonatal sepsis

I $\mathrm{t}$ is estimated that about 5 million neonates die every year in low-income countries. Infection contributes to approximately 30 to $40 \%$ of neonatal deaths in these countries ${ }^{1}$. However, early diagnosis of neonatal sepsis has remained a frustrating experience even in high-income countries ${ }^{2}$. This has prompted the evaluation of surrogate markers of inflammation as possible tools for early diagnosis of bacterial sepsis ${ }^{3-7}$.

\section{Correspondence to:}

Dr. Khaled Amro. Department of pediatric in Zarka military hospital. e.mail:drkhaledam64@yahoo.com
Estimations of cytokine levels and CRP levels are potentially useful in this respect ${ }^{3-8}$. Although several studies confirm that CRP levels are useful in the early diagnosis of sepsis, there are reports to the contrary ${ }^{9-12}$. It is suggested that serial rather than single determinations of CRP levels may be more useful in diagnosis of sepsis ${ }^{13}$. Such tests could be of special importance in a newborn that is asymptomatic or has only equivocal signs at birth but has risk factors for infection ${ }^{2}$.

The present study was designed to evaluate the effect of intrapartum risk factors for early onset sepsis (EOS) on neonatal CRP levels 
and the utility of CRP in the diagnosis of EOS.

\section{Methods}

This was a prospective cohort study conducted at a pediatric and obstetric department at military hospital in Zarka Jordan from March to October 2006.

\section{Inclusion and exclusion criteria}

Neonates were included if their mothers had at least one of the following risk factors for neonatal infection: prelabour rupture of membranes (ROM), more than three vaginal examinations after ROM, intrapartum fever (oral temperature $>38^{\circ} \mathrm{C}$ ), foul-smelling liquor, and untreated or partially treated urinary tract infection in the antenatal period. Newborn babies born at less than 28 weeks, weighing less than $1,000 \mathrm{~g}$ or with lethal congenital anomalies were excluded from the study.

\section{Primary outcome}

The primary outcome was EOS, defined as sepsis occurring within 48 hours of birth. The following were considered to be signs suggestive of sepsis: lethargy or poor feeding; axillary temperature $<36^{\circ} \mathrm{C}$ or $>38^{\circ} \mathrm{C}$ for more than one hour; significant jaundice with serum bilirubin $>15 \mathrm{mg} \%$ in the absence of blood group incompatibility; apnoea or respiratory distress; peripheral capillary refill time of $>3 \mathrm{sec}$ on the forehead or mid sternum; heart rate of $>160 / \mathrm{min}$ corrected for elevation of body temperature $\left(10\right.$ beats $/{ }^{\circ} \mathrm{C}$ rise); vomiting, diarrhoea or ileus; petechiae or bleeding diathesis; omphalitis; seizures. Laboratory markers considered abnormal were: total leukocyte count $<5,000 / \mathrm{mm} 3$, neutrophil count $<1,500 / \mathrm{mm} 3$, and immature to total neutrophil ratio $>0.2$.

Newborn babies developing signs suggestive of sepsis were categorized as having sepsis or probable sepsis. Sepsis was diagnosed if the newborn baby had signs suggestive of sepsis and a positive blood culture. Probable sepsis was diagnosed in a newborn baby with negative blood culture, if it had two or more signs suggestive of sepsis and one or more abnormal laboratory markers, or two or more abnormal laboratory markers with one or more signs suggestive of sepsis. Newborn babies with sepsis or probable sepsis received antibiotics for about 14 days. The remaining newborn babies were classified as at risk of infection and received antibiotics for an average of 5 days.

\section{Sample size estimation}

For an expected incidence of early onset sepsis among 4000 births of 3\% and a worst acceptable incidence of $1.5 \%$, the sample size required for $75 \%$ confidence is 184 . For an expected incidence of $2 \%$ and a worst acceptable incidence of $1 \%$, the sample size for $70 \%$ confidence is 182 . Therefore, a sample of 200 was studied.

\section{Laboratory techniques}

Approximately $3 \mathrm{~mL}$ of blood was collected from the umbilical cord after clamping and cutting of the cord. About $24 \mathrm{~h}$ later, approximately $2 \mathrm{~mL}$ of blood was collected by venepuncture from the new born. Samples were transported without delay to the laboratory for total leukocyte count, absolute neutrophil count, immature to total leukocyte ratio and CRP estimation. CRP levels were determined on a daily basis using a latex agglutination test (Omega Diagnostics Ltd, Alloa, Scotland, UK). This is a semiquantitative method with a detection limit of 6 $\mathrm{mg} / \mathrm{L}$. The investigator performing the CRP test was blinded to the clinical status of the newborn babies.

\section{Data collection and analyses}

Newborn babies were observed for signs of sepsis for at least $48 \mathrm{~h}$. Clinical data were collected using a questionnaire. Data were analyzed using EpiInfo Version 6. Proportions were compared by Chi-square test. Relative risks were calculated for the risk factors for sepsis. The predictive values of CRP for diagnosing neonatal sepsis were also calculated.

\section{Results}

There were 200 newborn babies enrolled for the study. The mean $\pm(\mathrm{SD})$ gestational age was $38.5 \pm(2.2)$ weeks. Seven (3.5\%) neonates had CRP levels of $\geq 6 \mathrm{mg} / \mathrm{L}$ in cord 
blood while 82 babies (41\%) had elevated levels at 24 hours. CRP levels in cord blood of $\geq 6 \mathrm{mg} / \mathrm{L}$ was significantly associated with rupture of membranes for more than 24 hours, labour for more than 12 hours and maternal fever. At 24 hours, elevation in CRP levels was significantly associated with primiparity, more than three vaginal examinations after rupture of membranes, meconium staining of amniotic fluid and amnioinfusion. When the cut-off CRP level was increased to $12 \mathrm{mg} / \mathrm{L}$, significant association was noted only with maternal fever. There was no association between Apgar score, birth weight and CRP levels.

Within 48 hours, 41 of the 200 babies with risk of infection developed at least one sign attributable to infection. Twenty seven had more than one sign. Of these, only two babies were diagnosed to have sepsis. Group B beta haemolytic streptococci were isolated from blood culture in one baby, while the other had coagulase-negative staphylococci. An additional eight babies were diagnosed to have probable sepsis. The sensitivity, specificity, positive and negative predictive values of CRP estimation at $24 \mathrm{hr}$ for diagnosis of EOS using $6 \mathrm{mg} / \mathrm{L}$ as the cut off were $80 \%, \quad 60 \%, \quad 7.7 \%$ and $98.6 \%$ respectively. The corresponding values for a cut off level of $12 \mathrm{mg} / \mathrm{L}$ were $30 \%, 81.3 \%$, $6.3 \%$ and $96.5 \%$ respectively.

Table 1 provides association between CRP levels and sepsis. CRP elevation was not significantly associated with the presence or number of signs. It was also noted that 10 of the 12 babies with CRP levels of $48 \mathrm{mg} / \mathrm{L}$ or more did not have evidence of infection. Only three of the 48 babies with CRP levels above $12 \mathrm{mg} / \mathrm{L}$ were diagnosed to have EOS.

Table 1:

CRP levels and neonatal sepsis.

\begin{tabular}{c|ccc}
$\begin{array}{c}\text { CRP levels } \\
(\mathrm{mg} / \mathrm{L})\end{array}$ & Sepsis & $\begin{array}{c}\text { Probable } \\
\text { sepsis }\end{array}$ & $\begin{array}{c}\text { No } \\
\text { sepsis }\end{array}$
\end{tabular}

Cord blood $<6(\mathbf{6}=\mathbf{1 9 5})$ At 24 hrs

\begin{tabular}{c|ccc}
$<6$ & 0 & 1 & 122 \\
6 & 1 & 3 & 34 \\
$>12$ & 1 & 2 & 31
\end{tabular}

Cord blood $>6 \quad(n=5)$ At 24 hrs

$<6$

6

$>12$
0

0

0
1

1

0
0

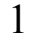

2
Only one baby among those with sepsis or probable sepsis had abnormal total leukocyte and absolute neutrophil counts in the cord blood. Five $(50 \%)$ had abnormal immature to total leukocyte ratio. Eighty nine of the 123 (72.3\%) CRP negative babies and 38 of the
82(46.34\%) CRP positive babies received antibiotics for less than three days.

\section{Discussion}

This study done to evaluate the association between intrapartum risk factors for infection 
with CRP levels showed that several such risk factors can cause elevated CRP levels in the absence of infection. This is in agreement with previously published reports ${ }^{7-13}$. Since CRP does not cross placenta, the elevated levels are due to production of CRP in the neonate. Chorioamnionitis can result in elevation of IL 6 levels even in uninfected neonates ${ }^{7}$. Stimuli other than infection, like hypoxia, trauma and metabolic changes can also induce production of proinflammatory mediators $^{7}$. Significant association is reported between birth asphyxia and elevated IL 6 levels. In prolonged labour, IL 6 levels rise in the neonate probably related to physical activity of labour. This cytokine stimulates CRP production.

There are few longitudinal studies examining CRP changes in healthy babies with intrapartum risk of infection. Cytokine elevation seen in the early neonatal period in such babies probably reflects physiological stress induced at birth ${ }^{13}$. Since CRP levels rise during the initial 24 hours in many babies irrespective of infection or administration of antibiotics, serial determinations in this period may not be of much use in diagnosis but may help in identifying uninfected babies and restricting antibiotic use ${ }^{14-15}$. Our data showed lower antibiotic use in babies who were CRP negative.

Various studies utilizing varying protocols have suggested different values as upper limit of normal ${ }^{8}$. In our study, at $24 \mathrm{~h}, \mathrm{CRP}$ levels of $6 \mathrm{mg} / \mathrm{L}$ had a negative predictive value of $99 \%$. This level therefore could be used to guide antibiotic therapy when latex agglutination kits are used. Testing samples in further dilutions to establish the actual amount of CRP may not be necessary since increasing levels were not associated with increasing severity or prognosis. Cord blood CRP levels estimated using a kit with $6 \mathrm{mg} / \mathrm{L}$ as detection limit, could not satisfactorily predict EOS. Recent studies show that cut off values may be different for cord and $24 \mathrm{~h}$ samples $^{7}$. More sensitive techniques like nephelometry may help set cut off levels for cord blood. In comparison to leukocyte counts and ratios, CRP levels at $24 \mathrm{~h}$ proved to be the single best indicator for diagnosing EOS. However, the $80 \%$ sensitivity obtained is unacceptably low for making critical decisions. If utilized with caution, this test can help in reducing antimicrobial use in the newborn.

\section{Conclusion}

Intrapartum risk factors for early onset sepsis can cause elevation of cord and neonatal CRP levels in the absence of infection. A CRP level of $<6 \mathrm{mg} / \mathrm{L}$ at $24 \mathrm{~h}$ has a good negative predictive value for neonatal sepsis. Serial CRP levels are not useful in diagnosing early onset sepsis.

\section{References}

1. The WHO young infants study group, Bacterial etiology of serious infections in young infants in developing countries: results of a multicentric study, Pediatr Infect Dis J 1999; 18: S17-22.

2. Escobar GJ. Effect of systemic inflammatory response on biochemical markers of neonatal bacterial infection: A fresh look at old confounders. Clini Chem 2003; 49: 21-22.

3. Ng PC, Cheng SH, Chui KM et al. Diagnosis of late onset neonatal sepsis with cytokines, adhesion molecule, and C-reactive protein in preterm very low birthweight infants. Arch Dis Child Fetal Neonatal Ed 1997; 77: F221-F227.

4. Chan DK, Ho LY. Usefulness of C-reactive protein in the diagnosis of neonatal sepsis. Singapore Med J 1997; 38: 252-255.

5. Magudumana MO, Ballot DE, Cooper PA et al. Serial interleukin 6 measurements in the early diagnosis of neonatal sepsis. J Trop Pediatr 2000; 46: 267-271.

6. Dollner H, Vatten L, Linnebo I et al. Inflammatory mediators in umbilical plasma from neonates who develop early-onset sepsis. Biol Neonate 2001; 80: 4147.

7. Chiesa C, Pellegrini G, Panero A et al. C-reactive protein, interleukin 6 and procalcitonin in immediate post natal period: influence of illness severity, risk status, antenatal and perinatal complications and infection. Clin Chem 2003; 49: 60 -68

8. Vesikari T. Cytokine determinations and rapid diagnosis of early onset neonatal septicemia. Acta Pediatr 1999; 88: 585-591.

9. Suri M, Thirupuram S, Sharma VK. Diagnostic and prognostic utility of C-reactive protein, alpha-1antitrypsin and alpha-2-macroglobulin in neonatal sepsis: a comparative account. Indian Pediatr 1991; 28 : 1159-1164.

10. Krediet T, Gerards L, Fleer A et al. The predictive value of CRP and I/T-ratio in neonatal infection. $J$ Perinat Med 1992; 20: 479-485. 
11. Anwer SK, Mustafa S. Rapid identification of neonatal sepsis. J Pak Med Assoc 2000; 50: 94-98.

12. Santana C, Guindeo MC, Gonzalez G et al. Cord blood levels of cytokines as predictors of early neonatal sepsis. Acta Pediatr 2001; 90: 1176-1181.

13. Chiesa C, Signore F, Assumma M et al. Serial measurements of C-reactive protein and interleukin-6 in the immediate postnatal period: reference intervals and analysis of maternal and perinatal confounders. Clin Chem 2001; 47: 1016-1022.
14. Philip AG, Mills PC. Use of C-reactive protein in minimizing antibiotic exposure: experience with infants initially admitted to a well-baby nursery. Pediatrics 2000; 106: E4.

15. Bomela HN, Ballot DE, Cory BJ et al. Use of Creactive protein to guide duration of empiric antibiotic therapy in suspected early neonatal sepsis. Pediatr Infect Dis J 2000;19: 531-535. 